\title{
Lytic Phase
}

National Cancer Institute

\section{Source}

National Cancer Institute. Lytic Phase. NCI Thesaurus. Code C19578.

A phase of the virus life cycle during which the virus replicates within the host cell, releasing a new generation of viruses when the infected cell lyses. 